
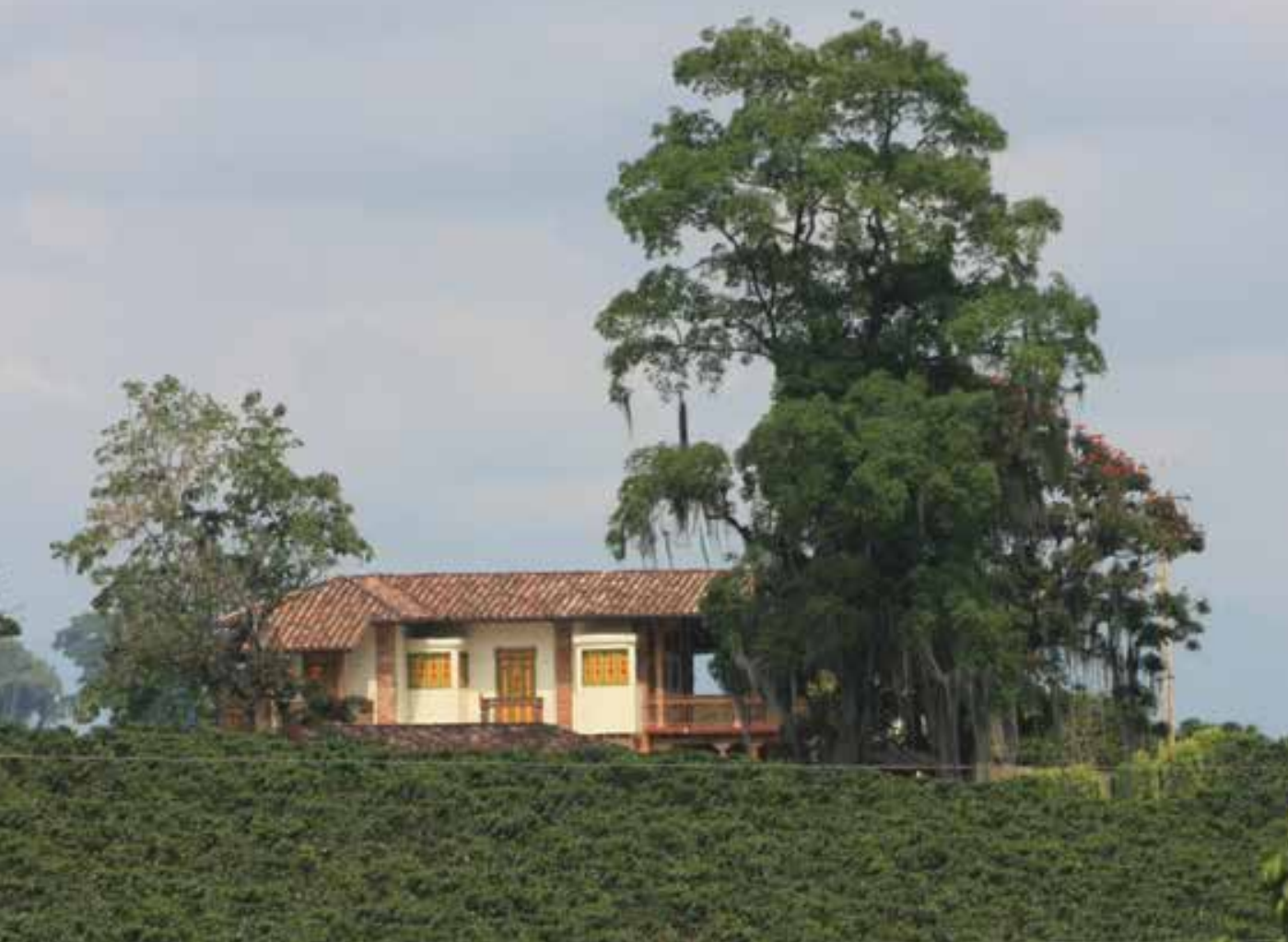

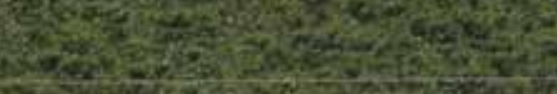

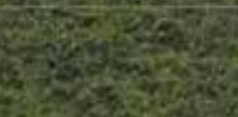$$
\text { Wrow }
$$

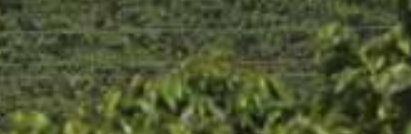

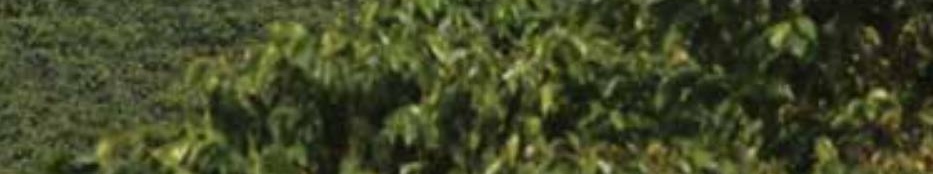$$
\log _{2} 62 x
$$

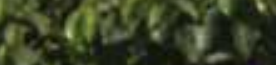$$
\text { trist. }
$$

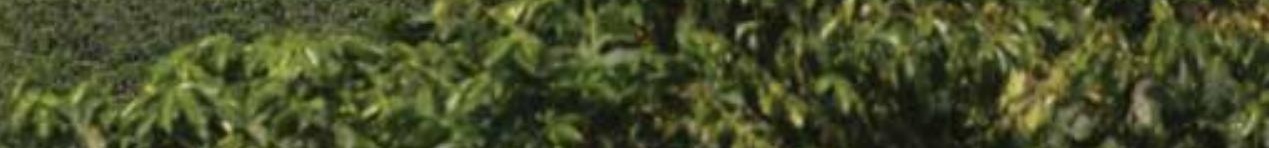

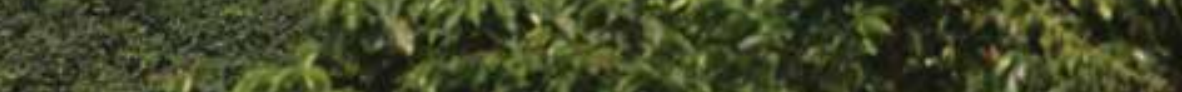

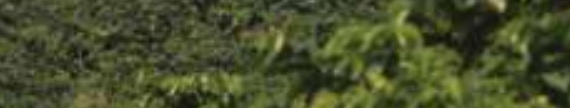

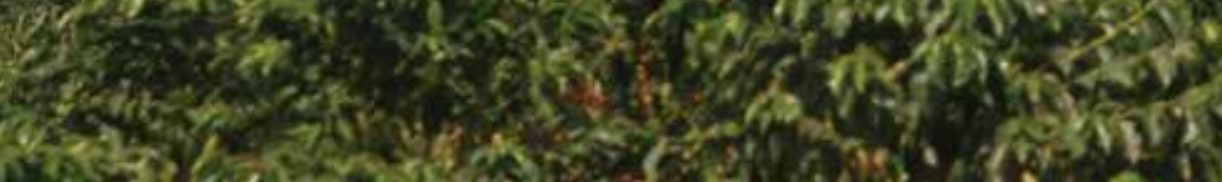

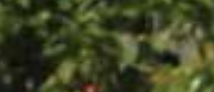

beitions
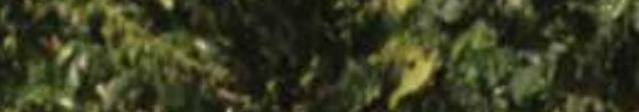

Flórez, C. P., Quiroga-Cardona, J., \& Arias, J. C. (2021). Variedades del Café. En Centro Nacional de

Investigaciones de Café, Guía más agronomía, más productividad, más calidad (3a ed., pp. 11-29). Cenicafé.

https://doi.org/10.38141/10791/0014_1

ORCID

Flórez, C. P. https://orcid.org/0000-0003-2859-3496

Quiroga-Cardona, J. https://orcid.org/0000-0002-2418-2318

Arias, J. C. https://orcid.org/0000-0002-5004-2423 


\section{VARIEDADES DE CAFÉ}

\section{Claudia Patricia Flórez Ramos * - Julio Quiroga Cardona ** - Juan Carlos Arias Suárez ***}

Siembre variedades mejoradas, resistentes a la roya del cafeto.

El Centro Nacional de Investigaciones de Café-Cenicafé optó por la estrategia de utilizar la diversidad genética como eje central para la obtención de variedades de café con resistencia durable a la roya del cafeto. Dentro de este concepto, las variedades desarrolladas por Cenicafé son compuestas, lo que significa que son la mezcla de diferentes progenies que difieren en su nivel de resistencia pero que comparten excelentes atributos agronómicos como producción, calidad física del grano y calidad sensorial.

En 1982, Cenicafé liberó la primera variedad resistente a la roya, en ausencia de la enfermedad: la variedad Colombia. Hoy, después de más de 30 años en los que esta variedad ha sido sembrada por los caficultores colombianos, continúa siendo resistente a la roya del cafeto, comprobando el éxito de la estrategia seleccionada por Cenicafé para el desarrollo de variedades mejoradas.

Bajo este mismo esquema, en el año 2002 entregó la variedad Tabi, recomendada para aquellas regiones con sistemas de producción tradicionales, donde son utilizadas variedades de porte alto. Tres años más tarde (2005) entregó a los caficultores la Variedad Castillo ${ }^{\circledR}$ con adaptación general a las condiciones de la caficultura colombiana, y sus siete componentes regionales (Pueblo Bello, El Rosario, Santa Bárbara, La Trinidad, Paraguaicito, Naranjal y El Tambo) adaptadas a regiones específicas de la caficultura del país. Estas variedades se 
destacan por tener en sus respectivos ambientes una productividad mayor a la variedad Castillo ${ }^{\circledR}$ General, porte intermedio, resistencia durable a la roya, tolerancia a la enfermedad de las cerezas del café (CBD) y alta calidad en taza.

En 2016, conscientes de los requerimientos de los caficultores colombianos, se liberó la variedad Cenicafé 1, que se caracteriza por presentar porte tipo Caturra, adaptación general a las condiciones de la zona cafetera colombiana, alta productividad, resistencia a la roya del cafeto y al CBD, y porcentaje de café supremo superior al de la Variedad Castillo ${ }^{\circledR}$. En las progenies componentes de la variedad Cenicafé 1 , el proceso de colonización y esporulación de la roya es reducido y demorado pero observable, por lo cual podrán verse niveles de afectación en el campo, pero sin que se llegue a ocasionar daño económico.

En 2017, teniendo presentes las evaluaciones históricas de incidencia y severidad por roya en las 48 progenies que conforman la Variedad Castillo ${ }^{\circledR}$ general y sus regionales, Cenicafé modificó su composición. De manera anticipatoria, sin que se haya presentado una afectación por la roya, Cenicafé entregó a los caficultores del país las variedades Castillo ${ }^{\circledR}$ zonales (zona Norte, zona Centro y zona Sur), que se caracterizan por presentar las mismas características agronómicas de la Variedad Castillo ${ }^{\circledR}$, en cuanto a mayor productividad, y con un mayor nivel de resistencia contra esta enfermedad. No obstante, al igual que Cenicafé 1 , en las selecciones de las variedades Castillo ${ }^{\circledR}$ zona Norte, zona Centro y zona Sur, los procesos de infección de la roya son reducidos y con tasas de esporulación lenta, pero que en el campo pueden llegar a ser visibles, sin que esto represente una afectación económica para el caficultor.

En términos generales, al ser una recomposición de la Variedad Castillo ${ }^{\circledR}$ y sus regionales, las variedades Castillo ${ }^{\circledR}$ zonales conservan las características agronómicas como producción, tamaño de grano, calidad en taza y porte.

A continuación, se describen las características de las variedades mejoradas Cenicafé 1 y las variedades Castillo ${ }^{\circledR}$ zona Norte, zona Centro y zona Sur. 


\begin{tabular}{|l|c|c|c|}
\multicolumn{1}{|c|}{ Variedad } & Progenies & $\begin{array}{c}\text { Rango de producción por } \\
\text { ciclo de cuatro cosechas } \\
\text { (kg de café cereza/árbol) }\end{array}$ & $\begin{array}{c}\text { Café } \\
\text { supremo } \\
(\%)\end{array}$ \\
\hline Cenicafé 1 & 8 & $16,7-18,6$ & $83,7-85,0$ \\
\hline Castillo ${ }^{\circ}$ zona Norte & 11 & $19,1-21,2$ & $75,9-78,6$ \\
\hline Castillo@ zona Centro & 11 & $20,2-22,1$ & $73,9-77,0$ \\
\hline Castillo@ zona Sur & 11 & $22,2-24,5$ & $78,5-81,0$
\end{tabular}

* Producción experimental con una densidad de 5.000 árboles/ha.

* Las variedades no superan los valores permitidos de granos vanos y caracol (<10\%).

A continuación, se describen las áreas de influencia de las variedades Castillo ${ }^{\circledR}$ zona Norte, zona Centro y zona Sur.

Todas las variedades desarrolladas que hacen parte del portafolio de la FNC están al servicio de los colombianos. Son variedades compuestas, conformadas por líneas mejoradas que no presentan necesariamente similitud en su apariencia, lo que significa que en el campo pueden observarse distintos tipos de arquitectura de planta y colores de brote (verde y bronce), sin que ello constituya una limitante en el desarrollo de su potencial agronómico y productivo.

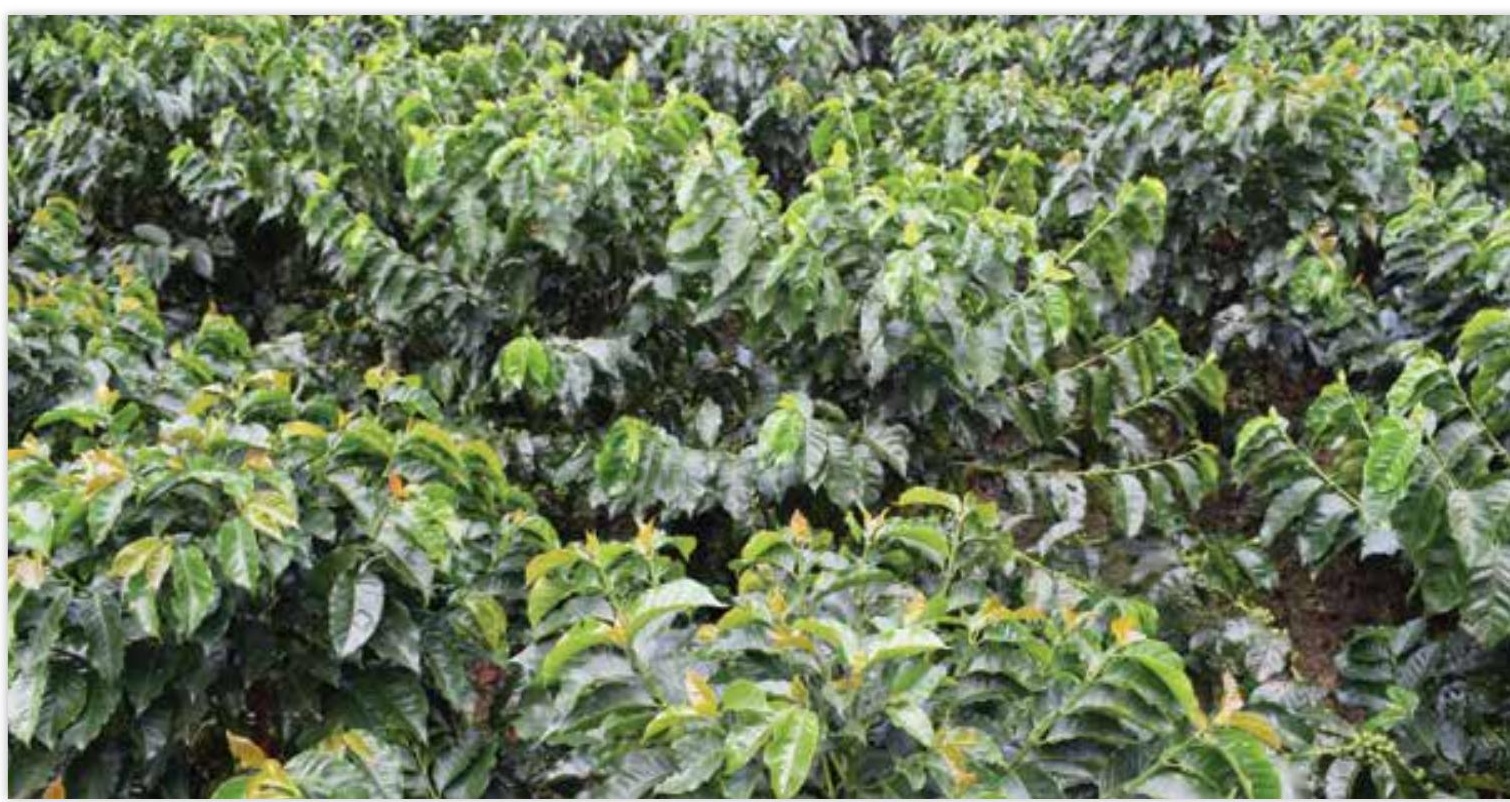




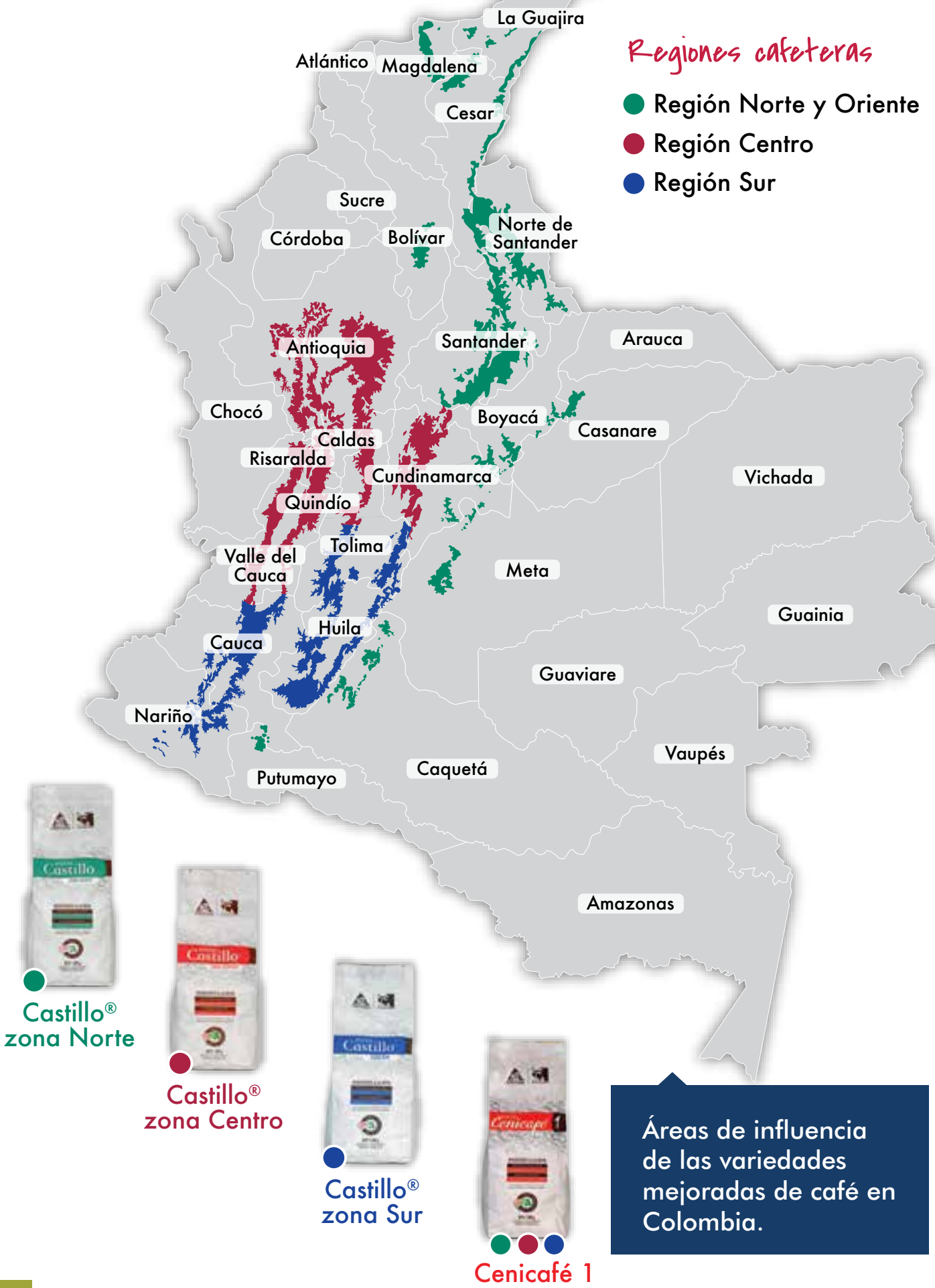




\begin{tabular}{|c|c|}
\hline \multicolumn{2}{|r|}{ Castillo $®$ zona Norte } \\
\hline Departamento & Municipios de influencia \\
\hline Bolívar & Morales, Santa Rosa del Sur. \\
\hline Boyacá & $\begin{array}{l}\text { Almeida, Berbeo, Campo Hermoso, Chinavita, } \\
\text { Chitaraque, Garagoa, Guateque, Guayatá, Labranza } \\
\text { Grande, Macanal, Miraflores, Moniquirá, Páez, } \\
\text { Pachavita, Pajarito, Paya, Pisba, Rondón, San Eduardo, } \\
\text { San José de Pare, San Luis de Gaceno, Santa María, } \\
\text { Santana, Somondoco, Togüí, Zetaquirá. }\end{array}$ \\
\hline Caquetá & $\begin{array}{l}\text { El Doncello, El Paujil, Florencia, Montañita, Puerto } \\
\text { Rico, San Vicente del Caguán. }\end{array}$ \\
\hline Casanare & $\begin{array}{l}\text { Chámeza, La Salina, Monterrey, Nunchía, Recetor, } \\
\text { Sácama, Támara, Tauramena, Yopal. }\end{array}$ \\
\hline Cesar & $\begin{array}{l}\text { Aguachica, Agustín Codazzi, Becerril, Chimichagua, } \\
\text { Chiriguaná, Curumaní, El Copey, González, La Gloria, } \\
\text { La Jagua de Ibirico, La Paz, Manaure Balcón del Cesar, } \\
\text { Pailitas, Pelaya, Pueblo Bello, Río de Oro, San Alberto, } \\
\text { San Martín, Valledupar. }\end{array}$ \\
\hline Cundinamarca & $\begin{array}{l}\text { Cáqueza, Choachí, Fómeque, Fosca, Gachalá, } \\
\text { Gachetá, Gama, Guayabetal, Gutiérrez, Junín, } \\
\text { Machetá, Manta, Medina, Paratebueno, Quetame, } \\
\text { Tibiritá, Ubalá, Ubaque. }\end{array}$ \\
\hline
\end{tabular}

Continúa... 


\begin{tabular}{|c|c|}
\hline La Guajira & $\begin{array}{l}\text { Barrancas, Dibulla, El Molino, Fonseca, Hato Nuevo, } \\
\text { La Jagua del Pilar, Riohacha, San Juan del Cesar, } \\
\text { Urumita, Villanueva. }\end{array}$ \\
\hline Magdalena & Aracataca, Ciénaga, Fundación, Santa Marta. \\
\hline Meta & $\begin{array}{l}\text { Acacias, Cubarral, Cumaral, El Castillo, El Dorado, } \\
\text { Lejanías, Mesetas, Restrepo, San Juan de Arama, } \\
\text { Villavicencio. }\end{array}$ \\
\hline $\begin{array}{l}\text { Norte de } \\
\text { Santander }\end{array}$ & $\begin{array}{l}\text { Ábrego, Arboledas, Bochalema, Bucarasica, Cáchira, } \\
\text { Cácota, Chinácota, Chitagá, Convención, Cúcuta, } \\
\text { Cucutilla, Durania, El Carmen, El Tarra, El Zulia, } \\
\text { Gramalote, Hacarí, Herrán, La Esperanza, La Playa, } \\
\text { Labateca, Los Patios, Lourdes, Ocaña, Pamplona, } \\
\text { Pamplonita, Ragonvalia, Salazar, San Calixto, San } \\
\text { Cayetano, Santiago, Sardinata, Teorama, Toledo, Villa } \\
\text { Caro, Villa Del Rosario. }\end{array}$ \\
\hline Putumayo & Mocoa. \\
\hline Santander & $\begin{array}{l}\text { Aguada, Albania, Aratoca, Barbosa, Barichara, } \\
\text { Betulia, Bolívar, Bucaramanga, Cabrera, Capitanejo, } \\
\text { Cepitá, Charalá, Charta, Chima, Chipatá, Confines, } \\
\text { Contratación, Coromoro, Curití, El Carmen de } \\
\text { Chucurí, El Guacamayo, El Peñón, El Playón, Encino, } \\
\text { Enciso, Florián, Floridablanca, Galán, Gámbita, Girón, } \\
\text { Guaca, Guadalupe, Guapotá, Guavatá, Güepsa, Hato, } \\
\text { Jesús María, Jordán, La Belleza, La Paz, Landázuri, } \\
\text { Lebrija, Los Santos, Málaga, Matanza, Mogotes, } \\
\text { Molagavita, Ocamonte, Oiba, Onzaga, Palmas del } \\
\text { Socorro, Páramo, Piedecuesta, Pinchote, Puente } \\
\text { Nacional, Rionegro, San Andrés, San Benito, San Gil, } \\
\text { San Joaquín, San José de Miranda, San Miguel, San } \\
\text { Vicente de Chucurí, Santa Bárbara, Santa Helena del } \\
\text { Opón, Simacota, Socorro, Suaita, Sucre, Suratá, Tona, } \\
\text { Valle De San José, Vélez, Villanueva, Zapatoca. }\end{array}$ \\
\hline
\end{tabular}


Castillo® zona Centro

\begin{tabular}{|c|c|}
\hline Departamento & Municipios de influencia \\
\hline Antioquia & $\begin{array}{l}\text { Abejorral, Abriaquí, Alejandría, Amagá, Amalfi, } \\
\text { Andes, Angelópolis, Angostura, Anorí, Anzá, } \\
\text { Argelia, Armenia, Barbosa, Bello, Betania, Betulia, } \\
\text { Briceño, Buriticá, Caicedo, Caldas, Campamento, } \\
\text { Cañasgordas, Caracolí, Caramanta, Carmen de } \\
\text { Viboral, Carolina, Cisneros, Ciudad Bolívar, Cocorná, } \\
\text { Concepción, Concordia, Copacabana, Dabeiba, Don } \\
\text { Matías, Ebéiico, Envigado, Fredonia, Frontino, Giraldo, } \\
\text { Girardota, Gómez Plata, Granada, Guadalupe, } \\
\text { Guatapé, Heliconia, Hispania, Itagüí, Ituango, } \\
\text { Jardín, Jericó, La Ceja, La Estrella, Liborina, Maceo, } \\
\text { Marinilla, Medellín, Montebello, Nariño, Olaya, Peñol, } \\
\text { Peque, Pueblo Rico, Remedios, Retiro, Sabanalarga, } \\
\text { Sabaneta, Salgar, San Andrés de Cuerquia, San } \\
\text { Carlos, San Francisco, San Jerónimo, San Luis, San } \\
\text { Rafael, San Roque, San Vicente, Santa Bárbara, } \\
\text { Santa Fe de Antioquia, Santa Rosa de Osos, Santo } \\
\text { Domingo, Sonsón, Sopetrán, Támesis, Tarso, Titiribí, } \\
\text { Toledo, Uramita, Urrao, Valdivia, Valparaiso, Vegachí, } \\
\text { Venecia, Yalí, Yarumal, Yolombó. }\end{array}$ \\
\hline Boyacá & $\begin{array}{l}\text { Briceño, Buenavista, Coper, La Victoria, Maripí, Muzo, } \\
\text { Otanche, Pauna, Quípama, San Pablo de Borbur, } \\
\text { Tununguá. }\end{array}$ \\
\hline Caldas & $\begin{array}{l}\text { Aguadas, Anserma, Aranzazu, Belalcázar, } \\
\text { Chinchiná, Filadelfia, La Merced, Manizales, } \\
\text { Manzanares, Marmato, Marquetalia, Marulanda, } \\
\text { Neira, Pácora, Palestina, Pensilvania, Riosucio, } \\
\text { Risaralda, Salamina, Samaná, San José, Supía, } \\
\text { Victoria, Villamaría, Viterbo. }\end{array}$ \\
\hline Chocó & El Carmen de Atrato. \\
\hline
\end{tabular}


Albán, Anapoima, Anolaima, Apulo, Arbeláez, Beltrán, Bituima, Cabrera, Cachipay, Caparrapí, Chaguaní, El Colegio, El Peñón, Fusagasugá, Guaduas, Guayabal de Síquima, Jerusalén, La Mesa, La Palma, La Peña, La Vega, Nilo, Nimaima, Nocaima, Pacho, Paime, Pandi,

Cundinamarca Pasca, Puli, Quebrada Negra, Quipile, San Antonio del Tequendama, San Bernardo, San Cayetano, San Francisco, San Juan de Río Seco, Sasaima, Silvania, Supatá, Tena, Tibacuy, Tocaima, Topaipí, Venecia, Vergara, Vianí, Villagómez, Villeta, Viotá, Yacopí, Zipacón.

\section{Quindío}

Armenia, Buenavista, Córdoba, Calarcá, Circasia, Filandia, Génova, La Tebaida, Montenegro, Pijao, Quimbaya, Salento.

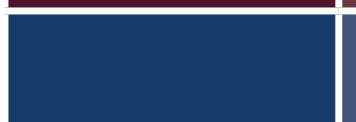

Apía, Balboa, Belén de Umbría, Dosquebradas, Risaralda Guática, La Celia, La Virginia, Marsella, Mistrató, Pereira, Pueblo Rico, Quinchía, Santa Rosa de Cabal, Santuario.

\section{Tolima}

Alvarado, Anzoátegui, Armero (Guayabal), Cajamarca, Casabianca, Falan, Fresno, Herveo, Ibagué, Lérida, Líbano, Mariquita, Murillo, Palocabildo, Santa Isabel, Venadillo, Villahermosa.

\section{Valle del Cauca}

Alcalá, Andalucía, Anserma Nuevo, Argelia, Bolívar, Buga, Bugalagrande, Caicedonia, Cali, Calima (EI Darién), Cartago, Dágua, El Águila, El Cairo, El Cerrito, El Dovio, Florida, Ginebra, Guacarí, Jamundí, La Cumbre, La Unión, La Victoria, Obando, Palmira, Pradera, Restrepo, Riofrío, Roldanillo, San Pedro, Sevilla, Toro, Truiillo, Tuluá, Ulloa, Versalles, Vijes, Yotoco, Yumbo. 


\begin{tabular}{|c|c|}
\hline \multicolumn{2}{|r|}{ Castillo ${ }^{\circledR}$ zona Sur } \\
\hline Departamento & Municipios de influencia \\
\hline Cauca & $\begin{array}{l}\text { Almaguer, Argelia, Balboa, Bolívar, Buenos Aires, } \\
\text { Caijibío, Caldono, Caloto, Corinto, El Tambo, } \\
\text { Florencia, Guachené, Inzá, Jambaló, La Sierra, } \\
\text { La Vega, Mercaderes, Miranda, Morales, Páez } \\
\text { (Belalcázar), Patía (El Bordo), Piendamó, Popayán, } \\
\text { Puracé, Rosas, San Sebastián, Santander de Quilichao, } \\
\text { Sotará (Paispamba), Suárez, Sucre, Timbío, Toribío, } \\
\text { Totoró. }\end{array}$ \\
\hline Huila & $\begin{array}{l}\text { Acevedo, Agrado, Aipe, Algeciras, Altamira, Baraya, } \\
\text { Campoalegre, Colombia, Elías, Garzón, Gigante, } \\
\text { Guadalupe, Hobo, Íquira, Isnos, La Argentina, La } \\
\text { Plata, Nátaga, Neiva, Oporapa, Paicol, Palermo, } \\
\text { Palestina, Pital, Pitalito, Rivera, Saladoblanco, San } \\
\text { Agustín, Santa María, Suaza, Tarqui, Tello, Teruel, } \\
\text { Tesalia, Timaná. }\end{array}$ \\
\hline Nariño & $\begin{array}{l}\text { Albán (San José), Ancuya, Arboleda (Berruecos), } \\
\text { Buesaco, Chachagüí, Colón (Génova), Consacá, } \\
\text { Cumbitara, El Peñol, El Rosario, El Tablón de Gómez, } \\
\text { El Tambo, Funes, Guaitarilla, lles, Imués, La Cruz, } \\
\text { La Florida, La Unión, Leiva, Linares, Los Andes } \\
\text { (Sotomayor), Mallama (Piedrancha), Pasto, Policarpa, } \\
\text { Providencia, Ricaurte, Samaniego, San Bernardo, San } \\
\text { Lorenzo, San Pablo, San Pedro de Carłago (Cartago), } \\
\text { Sandoná, Santa Cruz (Guachaves), Taminango, } \\
\text { Tangua, Túquerres, Yacuanquer. }\end{array}$ \\
\hline Tolima & $\begin{array}{l}\text { Alpujarra, Ataco, Chaparral, Coyaima, Cunday, } \\
\text { Dolores, Icononzo, Melgar, Natagaima, Ortega, } \\
\text { Planadas, Prado, Purificación, Rioblanco, Roncesvalles, } \\
\text { Rovira, San Antonio, San Luis, Suárez, Valle de San } \\
\text { Juan, Villarrica. }\end{array}$ \\
\hline
\end{tabular}


Aporte a la rentabilidad del cultivo de las variedades resistentes desarrolladas por cenicafé

La variedad de café hace parte de un complejo engranaje de prácticas agronómicas recomendadas para maximizar la rentabilidad del cultivo. Estas prácticas incluyen: la densidad de siembra, los ciclos de renovación, la fertilización, el sistema productivo (libre exposición/ sombra), el manejo de arvenses y el manejo fitosanitario, entre otras.

A continuación, se describen bajo diferentes escenarios de producción y favorabilidad para la roya, las pérdidas debidas a la no adopción de variedades de café resistentes a la enfermedad o su falta de manejo, cuando se utilizan variedades susceptibles.

Producción acumulada (@/ha de cps en un ciclo de cuatro cosechas)

\begin{tabular}{|c|c|c|}
\hline $\begin{array}{c}\text { Escenarios de } \\
\text { Producción }\end{array}$ & $\begin{array}{c}\text { Pérdida del potencial } \\
\text { productivo bajo escenario } \\
\text { climático desfavorable para } \\
\text { la roya }\left(23 \%{ }^{*}\right)\end{array}$ & $\begin{array}{c}\text { Pérdida del potencial } \\
\text { productivo bajo escenario } \\
\text { climático favorable para la } \\
\text { roya: año La Niña }\left(50 \%{ }^{*}\right)\end{array}$ \\
\hline 450 & $\$ 7.762 .500$ & $\$ 16.875 .000$ \\
\hline 600 & $\$ 10.350 .000$ & $\$ 22.500 .000$ \\
\hline 750 & $\$ 12.937 .500$ & $\$ 28.125 .000$ \\
\hline 900 & $\$ 15.525 .000$ & $\$ 33.750 .000$ \\
\hline 1050 & $\$ 18.112 .500$ & $\$ 39.375 .000$ \\
\hline
\end{tabular}

* Rivillas O.; C.A. Evolución de la roya del cafeto en plantaciones de café situadas en tres altitudes Informe Anual. Manizales: Cenicafé, 2015.

Sostenibilidad económica

A continuación, se relaciona el ahorro en los costos de producción por departamento cafetero, para los caficultores que han adoptado variedades resistentes a la roya. 
Costo del control de la roya (ha/año)* $\$ 750.000$

\begin{tabular}{|c|c|c|c|}
\hline \multirow[b]{2}{*}{ Departamentos } & \multicolumn{2}{|c|}{ Área cultivada en café (ha) } & \multirow{2}{*}{$\begin{array}{l}\text { Ahorro para el } \\
\text { Departamento }\end{array}$} \\
\hline & $\begin{array}{l}\text { Variedades } \\
\text { resistentes }\end{array}$ & $\begin{array}{l}\text { Variedades } \\
\text { susceptibles }\end{array}$ & \\
\hline Antioquia & $109.680,44$ & $12.363,43$ & 82,26 mil millones \\
\hline Bolívar & $1.121,53$ & 126,16 & 841,15 millones \\
\hline Boyacá & $7.274,44$ & $3.266,93$ & 5,45 mil millones \\
\hline Caldas & $56.908,99$ & $10.165,52$ & $42,68 \mathrm{mil}$ millones \\
\hline Caquetá & $2.988,24$ & $1.079,76$ & 2,24 mil millones \\
\hline Casanare & $2.430,21$ & 391,81 & $1,82 \mathrm{mil}$ millones \\
\hline Cauca & $75.792,95$ & $17.532,95$ & $56,84 \mathrm{mil}$ millones \\
\hline Cesar & $18.061,18$ & $8.522,94$ & 13,54 mil millones \\
\hline Chocó & 120,13 & 50,67 & 90,00 millones \\
\hline Cundinamarca & $27.512,99$ & $5.863,02$ & 20,63 mil millones \\
\hline Huila & $100.586,57$ & $46.688,87$ & 75,44 mil millones \\
\hline La Guajira & $2.379,65$ & $2.822,97$ & 1,78 mil millones \\
\hline Magdalena & $11.863,30$ & $7.496,15$ & $8,90 \mathrm{mil}$ millones \\
\hline Meta & $2.731,68$ & 561,16 & 2,05 mil millones \\
\hline Nariño & $30.072,46$ & $7.815,36$ & 22,5 mil millones \\
\hline Norte de Santander & $17.275,73$ & $6.213,06$ & 12,96 mil millones \\
\hline
\end{tabular}

Continúa... 
Costo del control de la roya (ha/año)* $\$ 750.000$

\begin{tabular}{|c|c|c|c|}
\hline \multirow[b]{2}{*}{ Departamentos } & \multicolumn{2}{|c|}{ Área cultivada en café (ha) } & \multirow{2}{*}{$\begin{array}{l}\text { Ahorro para el } \\
\text { Departamento }\end{array}$} \\
\hline & $\begin{array}{l}\text { Variedades } \\
\text { resistentes }\end{array}$ & $\begin{array}{l}\text { Variedades } \\
\text { susceptibles }\end{array}$ & \\
\hline Putumayo & 176,93 & 28,40 & 132,70 millones \\
\hline Quindío & $15.385,70$ & $6.613,52$ & 11,54 mil millones \\
\hline Risaralda & $39.075,95$ & 8. 165,05 & 29,30 mil millones \\
\hline Santander & $47.620,62$ & $2.164,85$ & $35,71 \mathrm{mil}$ millones \\
\hline Tolima & $86.263,38$ & $26.453,45$ & 64,70 mil millones \\
\hline Valle del Cauca & $44.354,34$ & $13.504,03$ & 33,26 mil millones \\
\hline Total Colombia & $699.677,41$ & $187.890,06$ & 525 mil millones \\
\hline
\end{tabular}

* Aplicación de cyproconazole (Alto 100 SL), tres aplicaciones por año utilizando una fumigadora de presión previa retenida.

Fuente: Sica, junio 2018.

Las variedades Cenicafé 1 y las variedades Castillo $®$ zona Norte, zona Centro y zona Sur, no requieren de la aplicación de fungicidas para el control de la roya, permitiendo la implementación de una caficultura rentable, sostenible y de calidad. 


\section{Recuerde que:}

$\rightarrow$ No hay variedades de café resistentes a la broca del café.

- Las variedades mejoradas desarrolladas por Cenicafé, son resistentes a la enfermedad de las cerezas del café (CBD), enfermedad restringida al continente africano, pero para la cual la caficultura colombiana ya está preparada.

- Los resultados de las pruebas de calidad en taza realizadas en las variedades desarrolladas por Cenicafé, indican que no existen diferencias en calidad con otras variedades de café como Típica y Borbón, reconocidas por la excelente calidad de la bebida.

- La única forma de garantizar la diversidad genética responsable de la resistencia estable y duradera a la roya, es mediante la adquisición de semilla certificada en los Comités de Cafeteros o Almacenes del Café de su localidad.

$\rightarrow$ Cada una de las variedades desarrolladas por Cenicafé son productos respaldados por más 20 años de investigación científica, que contribuyen a una caficultura rentable y sostenible.

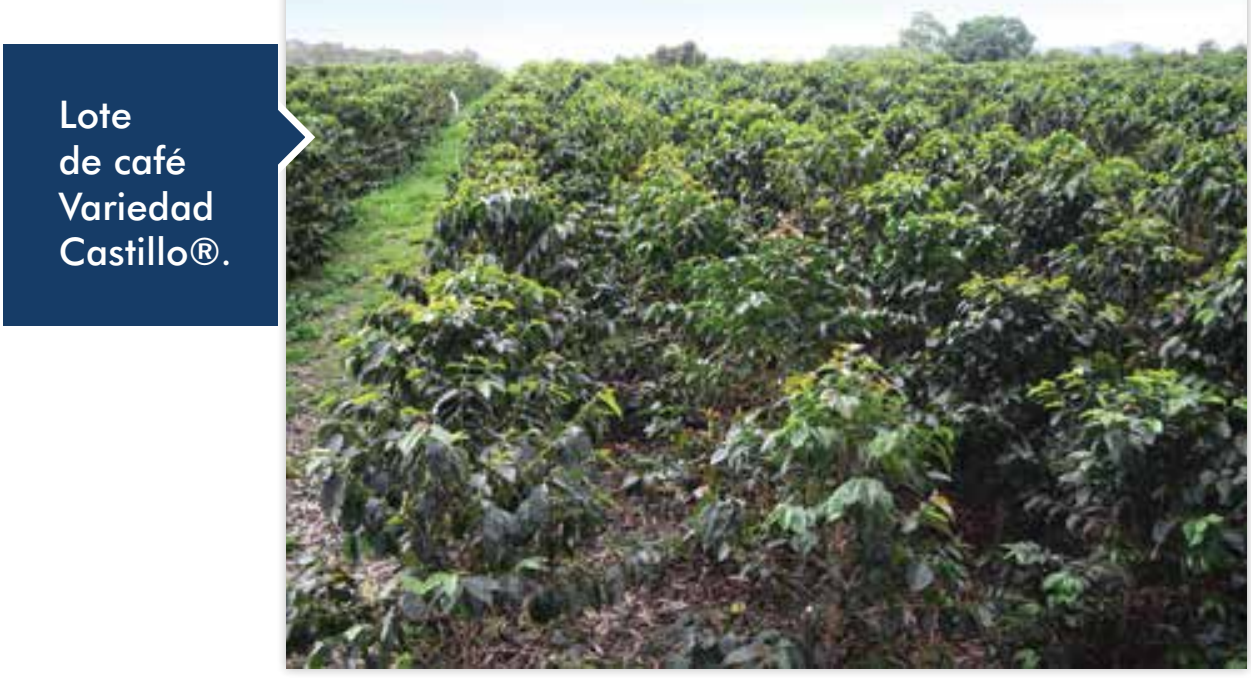




\section{Siembre variedades mejoradas, resistentes a la roya iel caitoto}
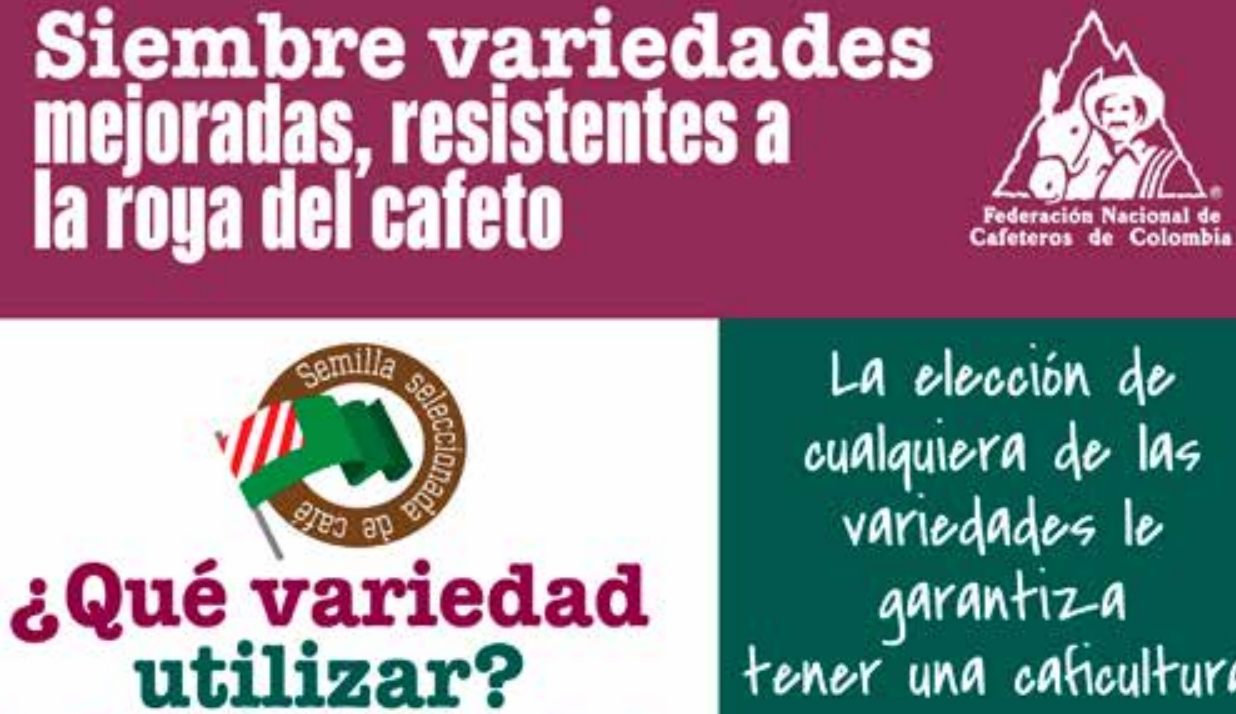

La elección de cualquiera de las variedades le garantiza tener una caficultura:

Castillo General, Castillo zona Norte ${ }^{\circledR}$, Castillo zona Centro ${ }^{\circledR}$,

Castillo zona Sur ${ }^{\circledR}$, Cenicafé 1 y Tabi La FNC tiene a su disposición un portafolio de variedades que se adaptan las necesidades de cada caficultor, todas con excelentes atributos agronómicos.

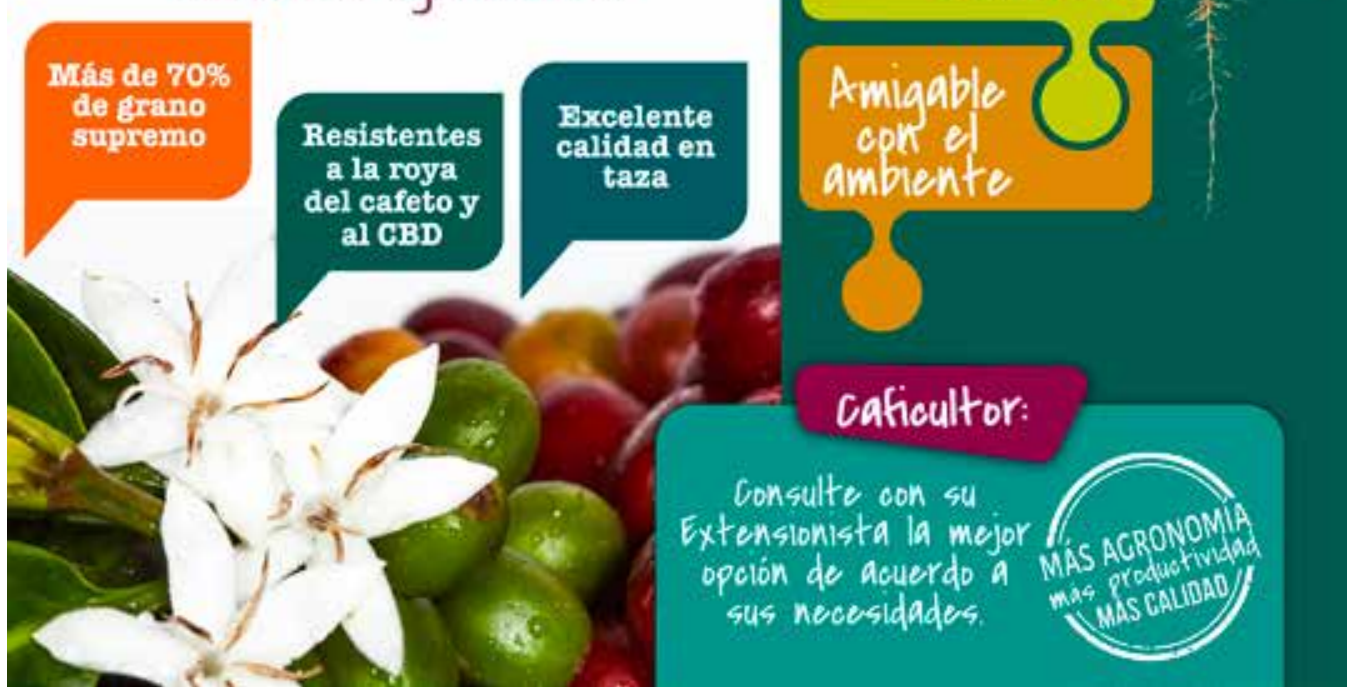


Siembre variedades mejoralias, resistentes a la roya ciel caleto

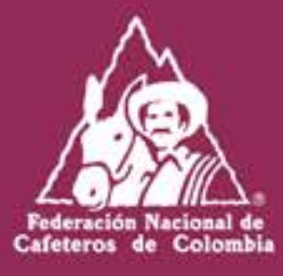

\section{Gastillo ${ }^{\circledR}$ General}

\section{Variedad desarrollada para los caficultores de Colombia}

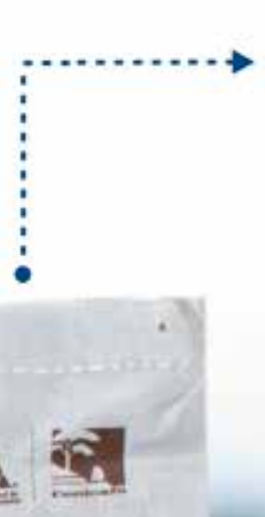

Resistente a la roya del cafeto

Resistente a la entermedad de las corezas del caketo (CBD)

Alto potencial de producción

Porte intermedio

Perfil de taza del café de Colombia

Castillo
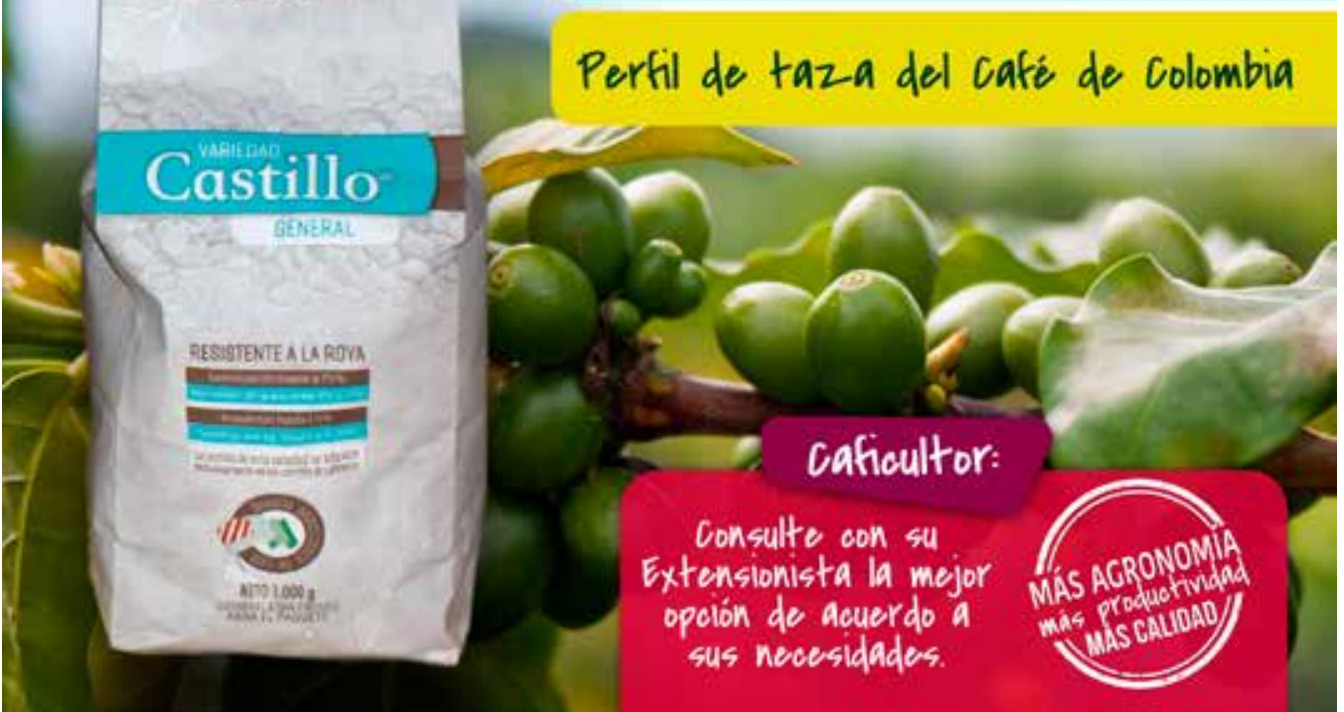
Siembre variedades mejoralias, resistentes a la roya ciel caleto

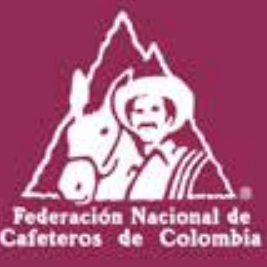

\section{Castillo ${ }^{\circledR}$ Zonales}
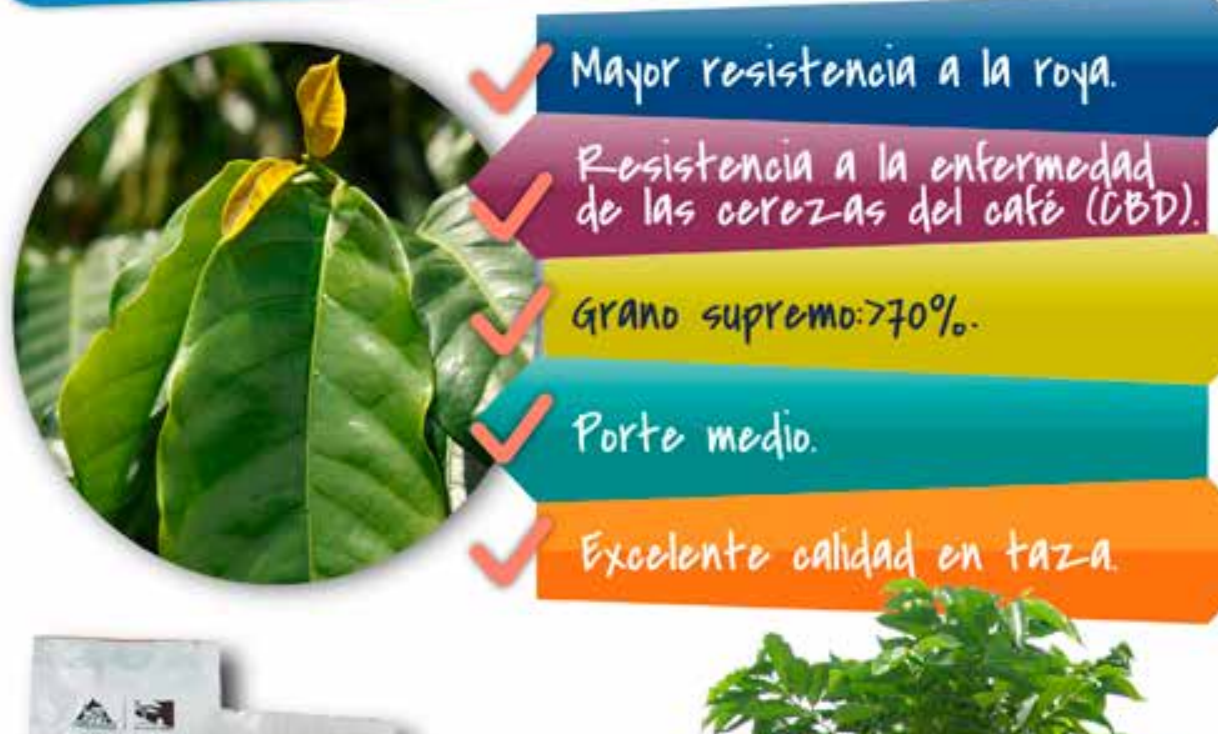

Castillo A i
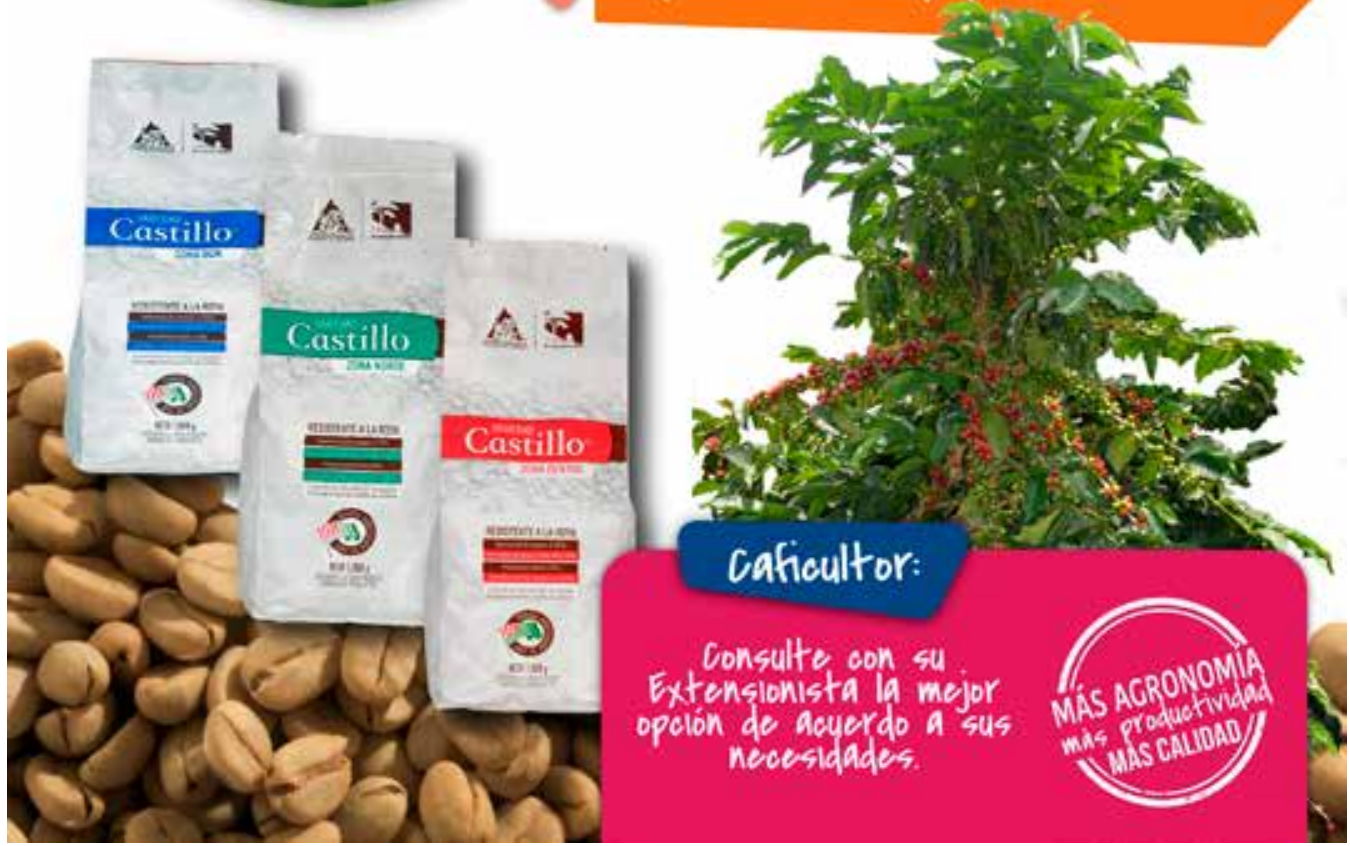


\section{Siembre variedades mejoraclas, resistentes a la roya ilet caleto}
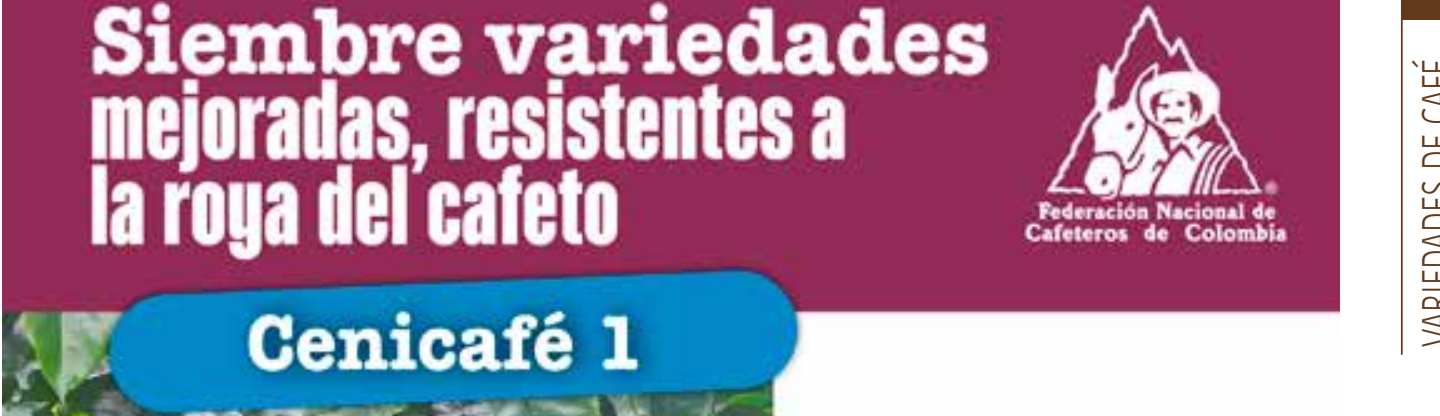

Porte tipo caturra

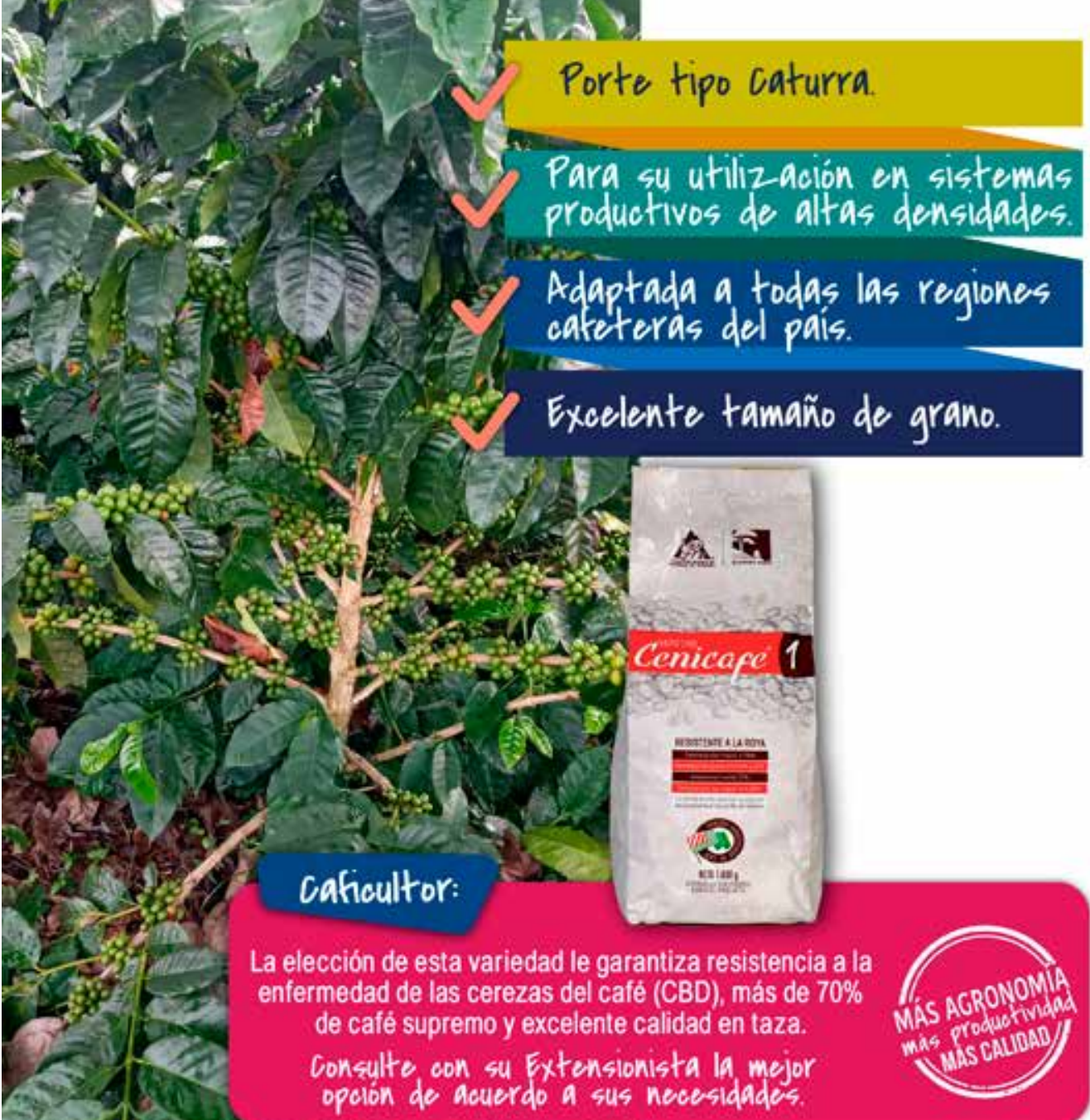


Siembre variedades mejoradias, resistentes a la roya ilet caitoto
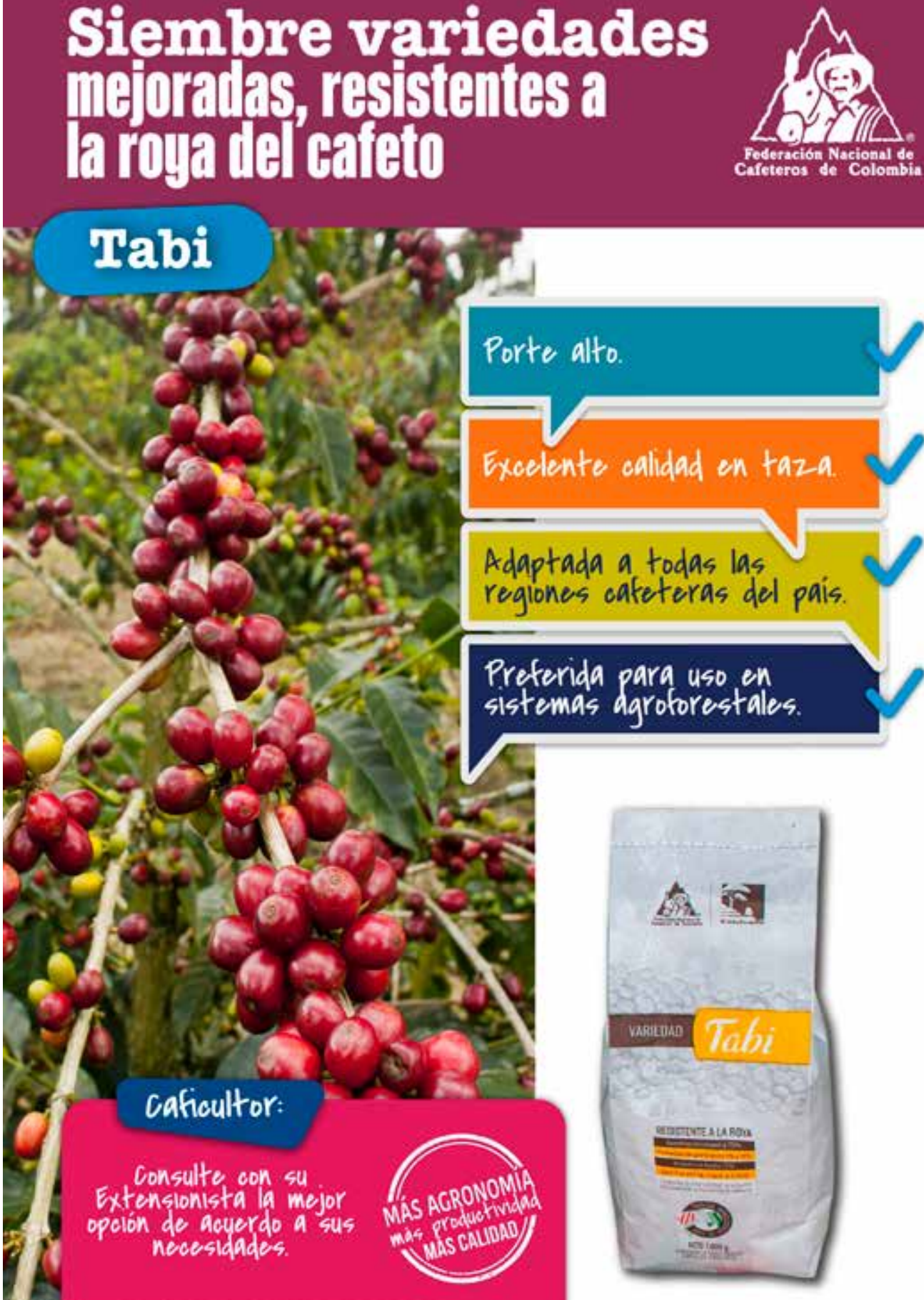


\section{Siembre variedades mejoradias, resistentes a la roya det caitoto

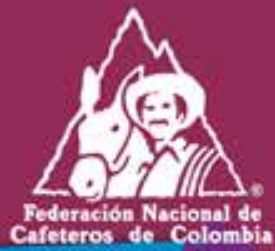 \\ Colección Golombiana de Gafe}

La colección busca conservar la variabilidad genética disponible para utilizarla en el desarrollo de nuevas variedades

\section{Más productivas}

Más resistentes

Con mayor calidad en taza

Adaptadas a la zona cafetera colombiana

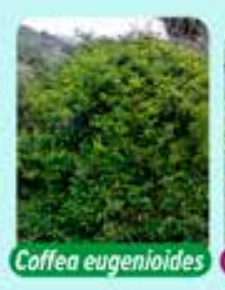

La existencia de la Colección ha permitido
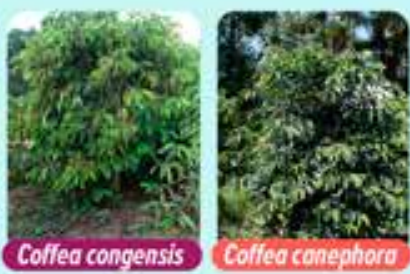

coffea coneahoro

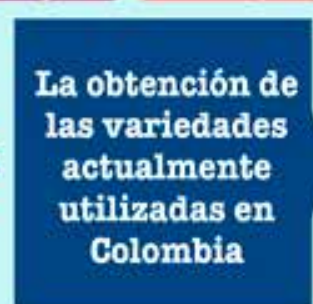

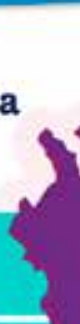
Cokrcion colombiona de cofe
CENCAFE-FWE

\section{Creada en 1947}

Genotipos diversos 1.031

\section{Especies de café 10} Materiales de Coffea arabica $85,26 \%$ 